\title{
FREE VIBRATION ANALYSIS OF BEAMS ON VARIABLE WINKLER ELASTIC FOUNDATION BY USING THE DIFFERENTIAL TRANSFORM METHOD
}

\author{
Alev Kacar, H. Tugba Tan, Metin O. Kaya \\ Faculty of Aeronautics and Astronautics \\ Istanbul Technical University, 34469, Maslak Istanbul, Turkey \\ kayam@itu.edu.tr
}

\begin{abstract}
In this study, free vibration of an Euler-Bernoulli beam resting on a variable Winkler foundation is considered. Structures which are supported along their length such as beams or pipelines resting on elastic soil are very commonly modeled with a Winkler foundation. In this problem, the elastic coefficient of the foundation is variable along the beam major axis. Constant, linear and parabolic variations are considered. The problem is handled for three different boundary conditions: simply supported-simply supported, clamped-clamped and cantilever (clamped-free) beams. The governing differential equations of the beam are solved by using Differential Transform Method (DTM). DTM is an easy transformation technique based on Taylor expansion series, providing high accuracy.
\end{abstract}

Key Words- Differential Transform Method, DTM, Elastic soil, Vibration, Beam, Pipeline

\section{INTRODUCTION}

During the past decades, various models have been introduced for beams resting on elastic foundations such as soil etc. Those structures supported along their main axis are represented by several approaches such as Winkler, Pasternak or Vlasov, Flonenko Borodich foundations. The Winkler modeling, one of the most fundamental methods was suggested in 1867 by Winkler. The approach introduces a linear algebraic relationship between the normal displacement of the structure and the contact pressure [1]. The Winkler Model represents the soil medium by a set of mutually independent spring elements. Such an approach grants simplicity in obtaining closed-form solutions $[2,3]$. Moreover, it gives the chance of obtaining a nonlinear behavior with lower computational effort compared to other methods [4-8].

There are numerous studies on the Winkler elastic foundation modeling in literature. Zhou [9] and Eisenberger [27] studied a general solution to vibrations of beams on a variable Winkler elastic foundation. Auersch [10] carried out a study about infinite beams on half-space compared with finite and infinite beams on a Winkler support. Eisenberger and Clastornik [11] examined the vibrations and buckling of a beam on a variable Winkler elastic foundation. Gupta et al. [12] presented buckling and vibrational behavior of polar orthotropic circular plates with linearly varying thickness. Also, Ruge and Birk [13] studied the dynamic behavior of infinite beam models, giving importance on asymptotic behavior at high frequencies. Dynamic response of a Timoshenko beam with a moving concentrated mass was solved by Lee [14]. Huang and Thambiratnam [15] who worked on the deflection of plates with moving accelerated 
loads by using Winkler model and the finite strip method. Oz and Pakdemirli has studied on resonances of shallow beams resting on elastic foundations [26]. Also, some researchers [16-17] studied the analysis of elastic foundations with Winkler-Pasternak models. In addition to differential transform method for structures on elastic foundation, Differential Quadrature Method (DQM) and Harmonic DQ methods are also widely used, where some of the studies of this method by Civalek [28-29] could be examined.

This study covers the free vibration of an Euler-Bernoulli beam resting on a variable Winkler elastic foundation. The elastic variation through the beam is handled in three cases: Constant, linear and parabolic variations. Boundary conditions of the beam are taken to be simply supported-simply supported, clamped-clamped and clamped-free ends, respectively. In order to find the natural frequencies, DTM is applied to the governing differential equations and boundary conditions. By using this method, these equations are transformed to a set of algebraic equations whose solutions give the desired results with an excellent accuracy compared with the exact results in open literature.

\section{EQUATION of MOTION and BOUNDARY CONDITIONS}

The governing differential equation of motion for an Euler-Bernoulli beam is expressed as follows;

$E I \frac{\partial^{4} w(x, t)}{\partial x^{4}}+\rho A \frac{\partial^{2} w(x, t)}{\partial t^{2}}+k(x) w(x, t)=0, \quad 0 \leq x \leq l$

where $l$ is the length, $E I$ is the bending rigidity, $\rho A$ is the mass per unit length, $k(x)$ is the elastic coefficient of Winkler foundation and $w(x, t)$ is the displacement.

Figure 1 represents the beam with constant cross-section laying on elastic Winkler foundation.

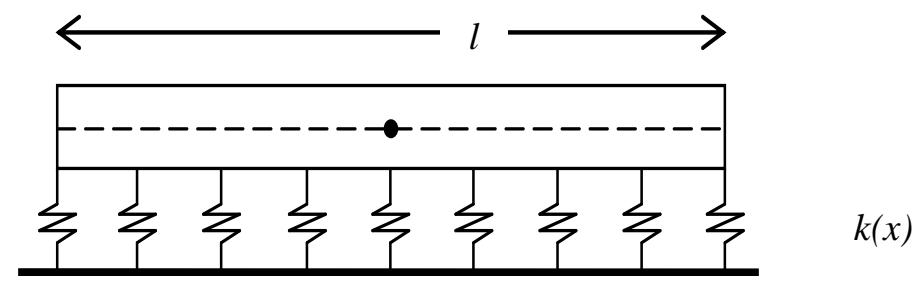

Figure 1: Beam structure resting on Winkler foundation

The relevant boundary conditions are;

$$
\begin{array}{lll}
\begin{array}{l}
\text { Simply supported-simply } \\
\text { supported: }
\end{array} & w=\frac{\partial^{2} w}{\partial x^{2}}=0 \text { at } x=0, l \\
\text { Clamped-clamped: } & w=\frac{\partial w}{\partial x}=0 \text { at } x=0, l \\
\text { Cantilevered: } & w=\frac{\partial w}{\partial x}=0 \text { at } x=0 \text { and } \frac{\partial^{2} w}{\partial x^{2}}=\frac{\partial^{3} w}{\partial x^{3}}=0 \text { at } x=l
\end{array}
$$

Assuming the displacement function as follows, where $\omega$ is the circular natural frequency; 
$w(x, t)=\bar{w}(x) e^{i \omega t}$

and substituting this into the governing differential equation Eq. 1 takes the form:

$E I \frac{\partial^{4} \bar{w}}{\partial x^{4}}-\rho A \omega^{2} \bar{w}+k(x) \bar{w}=0$

The elastic foundation is represented by a set of linear springs in Winkler modeling. In this study, Winkler elastic foundation can vary linearly or parabolically or even constantly through the length of the beam. Variation is given below for constant, linear and parabolic cases, respectively:

Constant:

$$
k(x)=k_{0}
$$

Linear:

$$
k(x)=k_{0}(1-\alpha x), \quad 0 \leq \alpha \leq 1
$$

Parabolic:

$$
k(x)=k_{0}\left(1-\beta x^{2}\right), \quad 0 \leq \beta \leq 1
$$

\section{NON-DIMENSIONALIZATION}

Defining a non-dimensional coordinate $\xi=\frac{x}{l}$, the equation of motion is obtained as;

$\frac{\partial^{4} \tilde{w}}{\partial \xi^{4}}+\left(K(\xi)-\lambda^{4}\right) \tilde{w}(\xi)=0$

Where dimensionless parameters are:

$\xi=\frac{x}{l} \tilde{w}=\frac{\bar{w}}{l} K(\xi)=\frac{k(\xi)}{E I} \lambda^{4} \quad \lambda^{4}=\frac{\rho A \omega^{2} l^{4}}{E I}$

Additionally, dimensionless boundary conditions can be expressed as follows;

Simply supported $\quad \tilde{w}=\frac{\partial^{2} \tilde{w}}{\partial \xi^{2}}=0$ at $\xi=0,1$

Clamped-clamped: $\quad \tilde{w}=\frac{\partial \tilde{w}}{\partial \xi}=0 \quad$ at $\xi=0,1$

Cantilever: $\quad \tilde{w}=\frac{\partial \tilde{w}}{\partial \xi}=0$ at $\xi=0$ and $\frac{\partial^{2} \tilde{w}}{\partial \xi^{2}}=\frac{\partial^{3} \tilde{w}}{\partial \xi^{3}}=0$ at $\xi=1$

and variation of elastic coefficient of Winkler foundation is expressed as;

Constant:

$$
K(\xi)=K_{0}
$$

Linear:

$$
K(\xi)=K_{0}(1-\alpha \xi)
$$

Parabolic:

$$
K(\xi)=K_{0}\left(1-\beta \xi^{2}\right)
$$

\section{DIFFERENTIAL TRANSFORM METHOD}

Differential transform method is an efficient technique for solving differential equations by iteration with considerable accuracy and easiness. It was first introduced by Zhou in 1986 [18], who applied differential transformation not only to linear but also to nonlinear initial value problems in electrical circuit analysis. Many scientists [19-24] have 
studied DTM in order to examine a variety of applications, such as the solution of nonlinear systems, eigen-value, and initial value or boundary value problems.

Differential transform is a simulation method which depends on Taylor Series expansion. The prime advantages of DTM, which make the method superior to others, are its accuracy, simplicity and rapidity. In contrast to higher order Taylor series method, DTM does not need the symbolic calculation of derivatives. It uses some transformation rules to transform original functions, including boundary conditions, into a set of algebraic functions [20]. Solving the algebraic set by iteration, desired results are obtained with great accuracy.

A function $f(x)$, analytical in domain $\mathrm{D}$ could be represented by a power series around any $\mathrm{x}=\mathrm{x}_{0}$ point in the current domain. The differential transform of the function $f(x)$ is given by

$F(r)=\frac{1}{r !}\left(\frac{d^{r} f(x)}{d x^{r}}\right)_{x=x_{0}}$

where $F(r)$ is the differentially transformed function and $\mathrm{r}$ is the member of the nonnegative integer domain $\mathrm{Z}^{+}$. Then the inverse transformation is described as;

$f(x)=\sum_{r=0}^{\infty}\left(x-x_{0}\right)^{r} F(r)$

Combining Eqs. 18 and 19, the following equation is obtained:

$f(x)=\sum_{r=0}^{\infty} \frac{\left(x-x_{0}\right)^{r}}{r !}\left(\frac{d^{r} f(x)}{d x^{r}}\right)_{x=x_{0}}$

Considering $f(x)$ by a series of finite terms, Eq. 20 is arranged as follows, with assuming the residual terms to be negligibly small. The increase of convergence is determined by the value $\mathrm{q}$.

$f(x)=\sum_{r=0}^{q} \frac{\left(x-x_{0}\right)^{r}}{r !}\left(\frac{d^{r} f(x)}{d x^{r}}\right)_{x=x_{0}}$

Basic transformation rules depending on the DTM for differential equations and boundary conditions are tabulated in Tables 1 and 2, respectively.

Table 2: Theorems of differential transform method for equation of motion

\begin{tabular}{ll}
\hline Original Function & Transformed Function \\
\hline$f(x)=g(x) \pm h(x)$ & $F(k)=G(k) \pm H(k)$ \\
$f(x)=\beta g(x)$ & $F(k)=\beta G(k)$ \\
$f(x)=g(x) h(x)$ & $F(k)=\sum_{l=0}^{k} G(l) h(k-l)$ \\
$f(x)=\frac{d^{n} g(x)}{d x^{n}}$ & $F(k)=\frac{(k+n) !}{k !} G(k+n)$
\end{tabular}




$$
f(x)=x^{n} \quad F(k)=\delta(k-n)=\left\{\begin{array}{l}
0 \text { if } k \neq n \\
1 \text { if } k=n
\end{array}\right.
$$

Table 3: Theorems of differential transform method for boundary conditions

\begin{tabular}{llll}
\hline \hline & $\boldsymbol{x}=\mathbf{0}$ & & $\boldsymbol{x}=\mathbf{1}$ \\
\hline Original B.C. & Transformed B.C. & Original B.C. & Transformed B.C. \\
\hline$f(0)=0$ & $F(0)=0$ & $f(1)=0$ & $\sum_{k=0}^{\infty} F(k)=0$ \\
$\frac{d f(0)}{d x}=0$ & $F(1)=0$ & $\frac{d f(1)}{d x}=0$ & $\sum_{k=0}^{\infty} k F(k)=0$ \\
$\frac{d^{2} f(0)}{d x^{2}}=0$ & $F(2)=0$ & $\frac{d^{2} f(1)}{d x^{2}}=0$ & $\sum_{k=0}^{\infty} k(k-1) F(k)=0$ \\
$\frac{d^{3} f(0)}{d x^{3}}=0$ & $F(3)=0$ & $\frac{d^{3} f(1)}{d x^{3}}=0$ & $\sum_{k=0}^{\infty} k(k-1)(k-2) F(k)$ \\
\hline \hline
\end{tabular}

\section{FORMULATION WITH DTM}

By using the DTM rules tabulated in Tables 1 and 2, below analytical expressions are obtained.

Constant modulus:

For constant elastic coefficient of Winkler foundation, $K(\xi)=K(0)$, the differential equation takes the form:

$\frac{d^{4} \tilde{w}}{d \xi^{4}}+\left(K_{0}-\lambda^{4}\right) \tilde{w}=0$

Applying DTM to the above equation, following recurrence relation is obtained:

$W(r+4)=\frac{\lambda^{4}-K_{0}}{(r+4)(r+3)(r+2)(r+1)} W(r)$

Linear modulus:

$\frac{d^{4} \tilde{w}}{d \xi^{4}}+\left(K_{0}-\lambda^{4}\right) \tilde{w}-\alpha K_{0} \xi \tilde{w}=0$

$W(r+4)=\frac{\lambda^{4}-K_{0}}{(r+4)(r+3)(r+2)(r+1)}\left(W(r)+\alpha K_{0} W(r-1)\right), \quad r \geq 1$

Parabolic variation:

$\frac{d^{4} \tilde{w}}{d \xi^{4}}+\left(K_{0}-\lambda^{4}\right) \tilde{w}-\beta K_{0} \xi^{2} \tilde{w}=0$

$W(r+4)=\frac{\lambda^{4}-K_{0}}{(r+4)(r+3)(r+2)(r+1)} W(r)+\frac{\beta W(r-2)}{(r+4)(r+3)(r+2)(r+1)}, \quad r \geq 2$ 
For example, $W(0)$ and $W(2)$ coefficients of the beam with both ends simply supported, are obtained by using boundary conditions, and $W(1)$ and $W(3)$ are set to unknown constants, namely;

$W(0)=0, W(1)=c_{1}, W(2)=0, W(3)=c_{2}$

Hereby, the calculation procedure is given only for parabolic variation. Using Eq. 27 and Eq. 28, $W(r)$ 's are evaluated in terms of $\lambda, K_{0}, c_{1}$ and $c_{2}$.

$W(4)=0$

$W(5)=\frac{\left(\lambda_{4}-K_{0}\right) c_{1}}{240}$

$W(6)=0$

$W(7)=\frac{\left(\lambda_{4}-K_{0}\right) c_{2}}{840}+\frac{\beta c_{1}}{840}$

Similar procedure is followed for the other two boundary conditions; clamped-clamped and cantilever beam.

In order to give an idea to the reader about the accuracy, free vibration case of an EulerBernoulli beam without foundation is compared to the Reference [25]. The Winkler elastic parameter is set to zero and the above DTM equations are ran. The results are given below:

Table 1: Comparison of DTM with exact solutions

\begin{tabular}{ccl}
\hline \hline Mode & \multicolumn{1}{c}{ DTM } & \multicolumn{1}{c}{$[25]$} \\
\hline 1 & 1.875104069 & 1.8751014 \\
2 & 4.694091133 & 4.694091 \\
3 & 7.854757438 & 7.854757 \\
4 & 10.99554073 & 10.995541 \\
\hline \hline
\end{tabular}

As seen from the above table, differential transform method provides the satisfying accuracy. The method also is also accurate for the free vibrational modes of the beam lying on Winkler foundation, which is the main subject of the study and where the results are given with comparison to the open literature in the Tables 2-7. Through the study, 50 iteration has been used and for the solutions.

The convergence of the first six natural frequency set is introduced at Figure 2. At least 55 terms should be evaluated for five-digit precision for the 6th natural frequency. By definition of differential transform method, as the higher terms are evaluated, the more natural frequencies are obtained. 


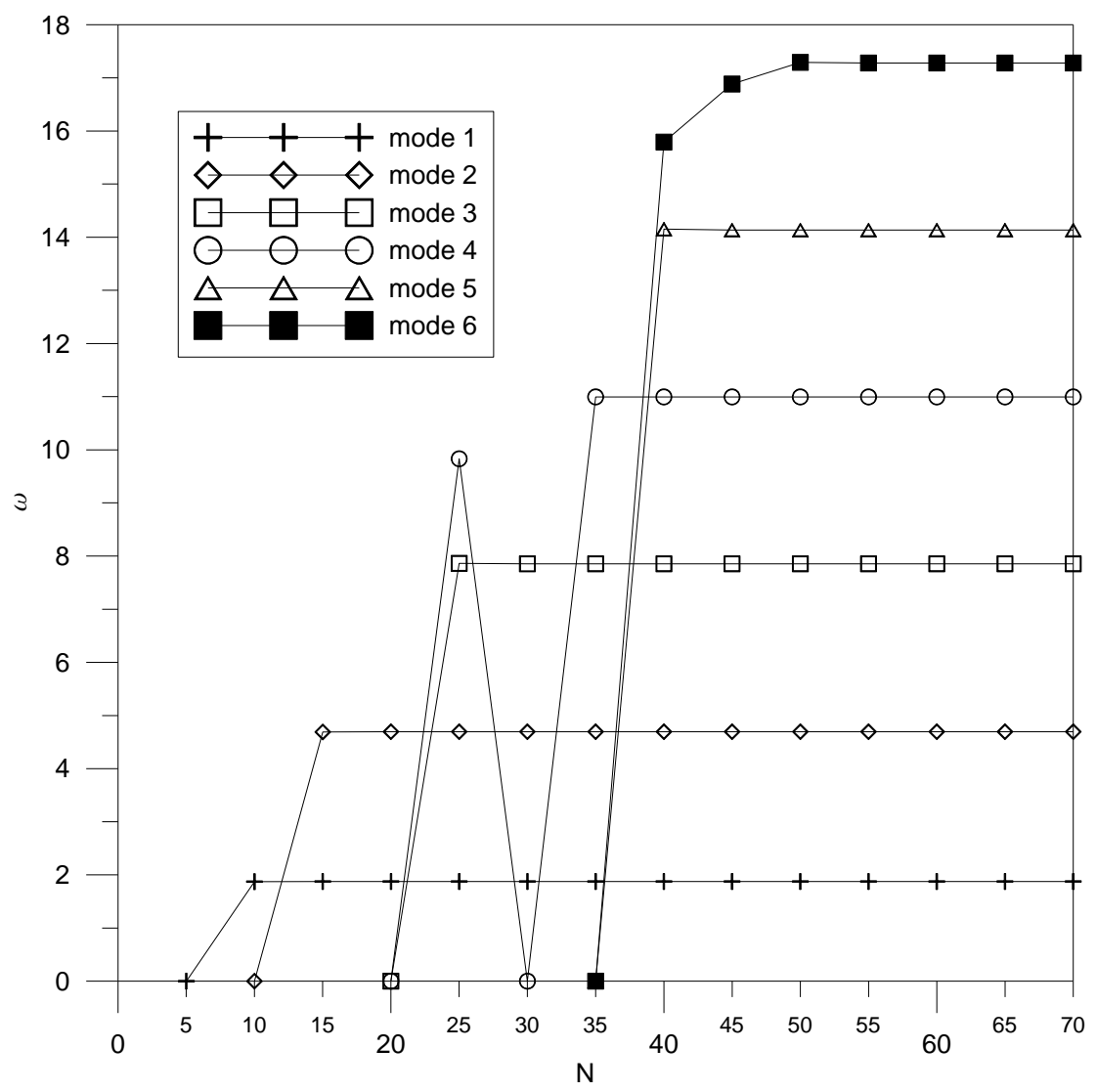

Figure 2: Convergence of natural frequencies with $\mathrm{N}$

\section{NUMERICAL RESULTS}

Frequency parameters $\lambda i$ for different boundary conditions are tabulated in this section. In particular the results of the simply supported-simply supported case at different K0 values are given extensively. The present results are compared with various results in open literature.

\subsection{Simply Supported-Simply Supported Beam}

Frequency variation for simply supported-simply supported beam at constant elastic, linear and parabolic modulus is shown at Figure 3, 4 and 5, respectively.
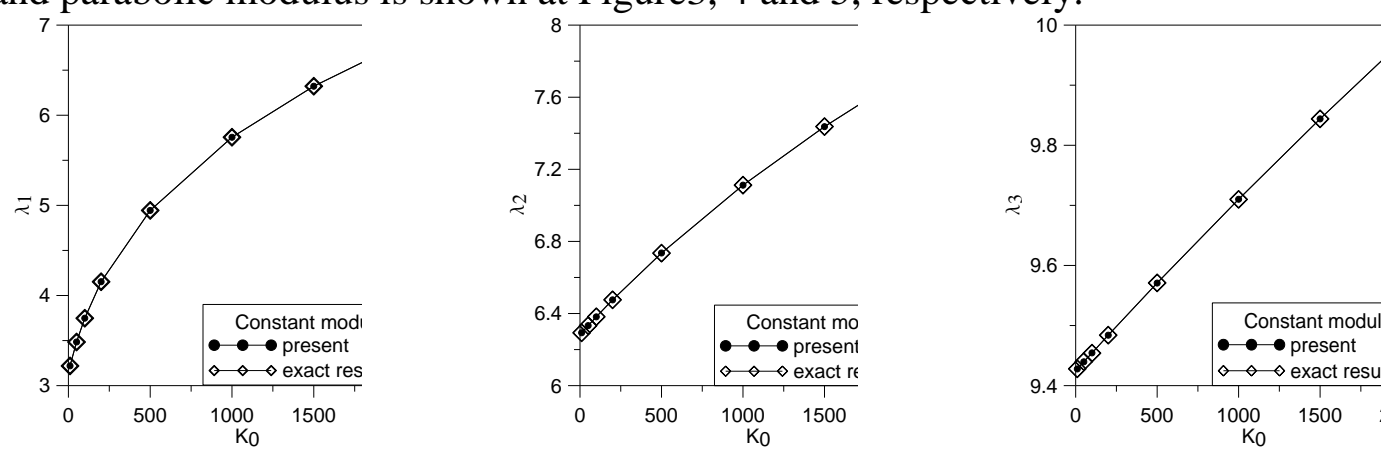

Figure 3: Frequency variation of S-S beam with constant modulus 

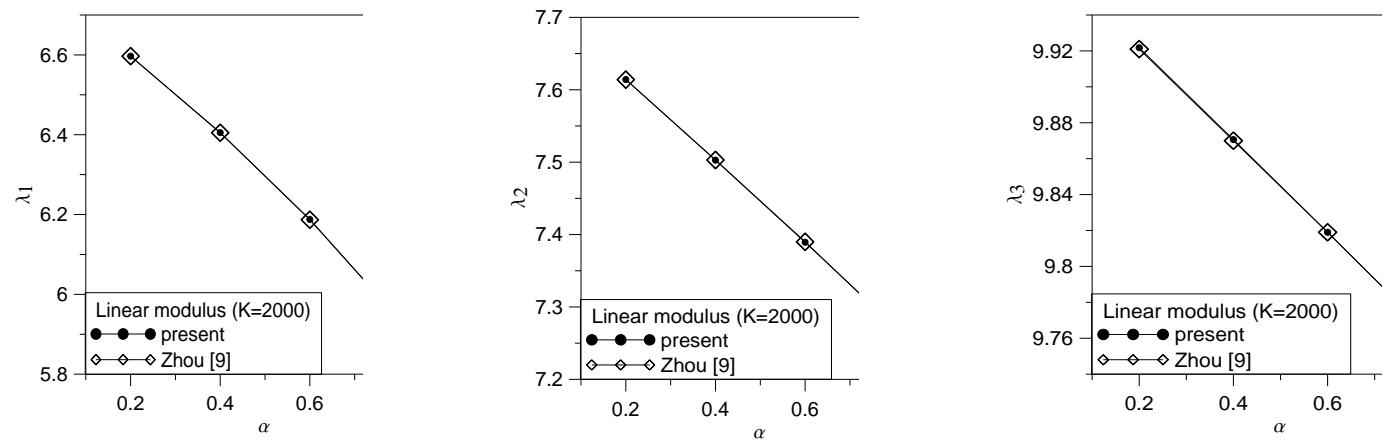

Figure 4: Frequency variation of S-S beam with linear modulus
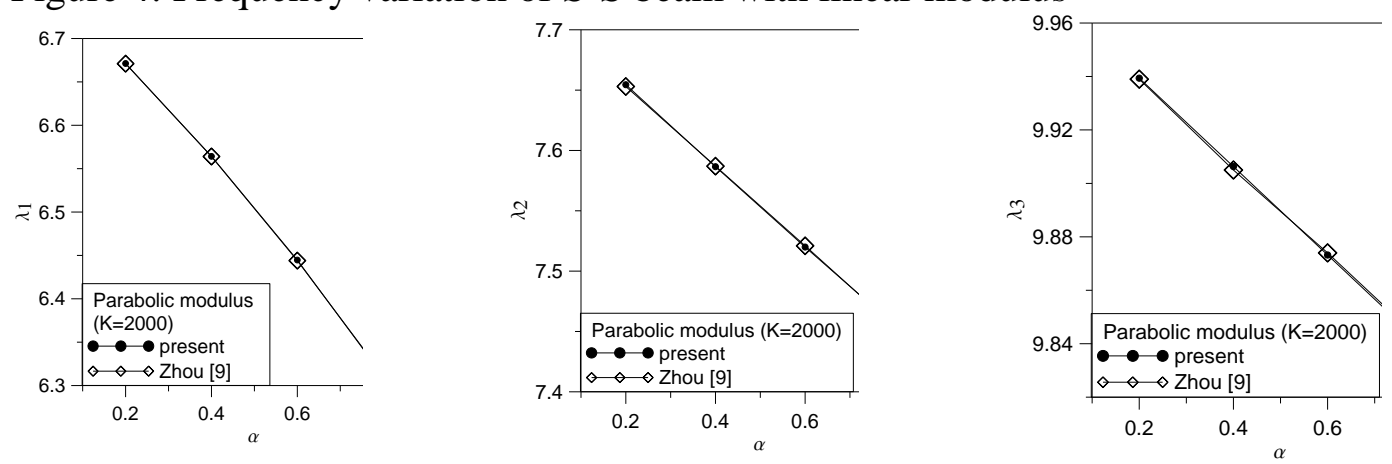

Figure 5: Frequency variation of S-S beam with parabolic modulus

Frequency parameters for above conditions are listed at Table 4, 5, and6, respectively.

Table 4: Frequency parameters for S-S beam at constant elastic modulus

\begin{tabular}{ccccccccc}
\hline \hline $\mathbf{K}_{\mathbf{0}}$ & $\boldsymbol{\lambda}_{\mathbf{1}}$ & $\boldsymbol{\lambda}_{\mathbf{2}}$ & $\boldsymbol{\lambda}_{\mathbf{3}}$ & $\boldsymbol{\lambda}_{\mathbf{4}}$ & $\boldsymbol{\lambda}_{\mathbf{5}}$ & $\boldsymbol{\lambda}_{\mathbf{6}}$ & $\boldsymbol{\lambda}_{\mathbf{7}}$ & $\boldsymbol{\lambda}_{\mathbf{8}}$ \\
\hline $\mathbf{1 0}$ & 3,219291184 & 6,293239752 & 9,427762796 & 12,56763025 & 15,70860826 & 18,84992919 & 21,99138364 & 25,13289871 \\
& $(3,219)^{*}$ & $(6,293)$ & $(9,428)$ & $(12,568)$ & $(15,709)$ & $(18,850)$ & $(21,991)$ & $(25,133)$ \\
$\mathbf{5 0}$ & 3,484424567 & 6,33298318 & 9,439673875 & 12,57266501 & 15,71118743 & 18,85142205 & 21,99232383 & 25,13352858 \\
& $(3,484)$ & $(6,333)$ & $(9,440)$ & $(12,573)$ & $(15,711)$ & $(18,851)$ & 21,992 & $(25,134)$ \\
$\mathbf{1 0 0}$ & 3,748364250 & 6,381633293 & 9,454499603 & 12,57894997 & 15,71440961 & 18,85328763 & 21,99349889 & 25,13431586 \\
& $(3,748)$ & 6,382 & $(9,454)$ & $(12,579)$ & 15,714 & 18,853 & $(21,993)$ & $(25,134)$ \\
$\mathbf{2 0 0}$ & 4,152776516 & 6,475725032 & 9,483943557 & 12,59149170 & 15,72084802 & 18,85701712 & 21,99584846 & 25,13589020 \\
& $(4,153)$ & 6,476 & $(9,484)$ & $(12,591)$ & $(15,721)$ & $(18,857)$ & $(21,996)$ & 25,136 \\
$\mathbf{5 0 0}$ & 4,943880409 & 6,735814452 & 9,570668085 & 12,62889372 & 15,74011595 & 18,86819235 & 22,00289264 & 25,14061144 \\
& $(4,944)$ & $(6,736)$ & $(9,571)$ & $(12,629)$ & $(15,740)$ & $(18,868)$ & $(22,003)$ & $(25,141)$ \\
$\mathbf{1 0 0 0}$ & 5,755620336 & 7,112107040 & 9,710176091 & 12,69050177 & 15,77207279 & 18,88677371 & 22,01461793 & 25,14847427 \\
& $(5,756)$ & 7,112 & $(9,710)$ & 12,691 & $(15,772)$ & $(18,887)$ & $(22,015)$ & 25,149 \\
$\mathbf{1 5 0 0}$ & 6,321993397 & 7,436673846 & 9,843917717 & 12,75122540 & 15,80383655 & 18,90530040 & 22,02632451 & 25,15632973 \\
& $(6,322)$ & 7,437 & $(9,844)$ & $(12,751)$ & 15,804 & $(18,905)$ & $(22,026)$ & $(25,156)$ \\
$\mathbf{2 0 0 0}$ & 6,767383474 & 7,723570755 & 9,972420206 & 12,81109369 & 15,83540994 & 18,92377278 & 22,03801246 & 25,16417784 \\
& $(6,767)$ & $(7,724)$ & $(9,972)$ & $(12,811)$ & $(15,835)$ & $(18,924)$ & $(22,038)$ & $(25,164)$ \\
\hline \hline
\end{tabular}

* Exact results [9] 
Table 5: Frequency parameters for S-S beam at linear elastic modulus

\begin{tabular}{ccccccccc}
\hline \hline $\begin{array}{c}\alpha=0,2 \\
\mathbf{K}_{\mathbf{0}}\end{array}$ & $\boldsymbol{\lambda}_{\mathbf{1}}$ & $\boldsymbol{\lambda}_{\mathbf{2}}$ & $\boldsymbol{\lambda}_{\mathbf{3}}$ & $\boldsymbol{\lambda}_{\mathbf{4}}$ & $\boldsymbol{\lambda}_{\mathbf{5}}$ & $\boldsymbol{\lambda}_{\mathbf{6}}$ & $\boldsymbol{\lambda}_{\mathbf{7}}$ & $\boldsymbol{\lambda}_{\mathbf{8}}$ \\
\hline $\mathbf{1 0}$ & 3,211771150 & 6,292236540 & 9,427464444 & 12,56750430 & 15,70854376 & 18,84989187 & 21,99136013 & 25,13288352 \\
$\mathbf{5 0}$ & 3,454480416 & 6,328057675 & 9,438187563 & 12,57203601 & 15,71086511 & 18,85123547 & 21,99220627 & 25,13345065 \\
$\mathbf{1 0 0}$ & 3,699921549 & 6,371998414 & 9,451540469 & 12,57769379 & 15,71376534 & 18,85291457 & 21,99326387 & 25,13415880 \\
$\mathbf{2 0 0}$ & 4,080996862 & 6,457257919 & 9,478078411 & 12,58898659 & 15,71956102 & 18,85627142 & 21,99537858 & 25,13557555 \\
$\mathbf{5 0 0}$ & 4,836530578 & 6,694670148 & 9,556387790 & 12,62268461 & 15,73690986 & 18,86633128 & 22,00171909 & 25,13982521 \\
$\mathbf{1 0 0 0}$ & 5,618515814 & 7,042035261 & 9,682794448 & 12,67825778 & 15,76569823 & 18,88306219 & 22,01227444 & 25,14690373 \\
$\mathbf{1 5 0 0}$ & 6,165920336 & 7,344688366 & 9,804455230 & 12,73311253 & 15,79433039 & 18,89974892 & 22,02281476 & 25,15397413 \\
$\mathbf{2 0 0 0}$ & 6,596856775 & 7,614150729 & 9,921767296 & 12,78727024 & 15,82280832 & 18,91639176 & 22,03334004 & 25,16103879 \\
\hline \hline
\end{tabular}

Table 6: Frequency parameters for S-S beam at parabolic elastic modulus

\begin{tabular}{ccccccccc}
\hline \hline $\begin{array}{c}\mathbf{B}=0.2 \\
\mathbf{K}_{\mathbf{0}}\end{array}$ & $\boldsymbol{\lambda}_{\mathbf{1}}$ & $\boldsymbol{\lambda}_{\mathbf{2}}$ & $\boldsymbol{\lambda}_{\mathbf{3}}$ & $\boldsymbol{\lambda}_{\mathbf{4}}$ & $\boldsymbol{\lambda}_{\mathbf{5}}$ & $\boldsymbol{\lambda}_{\mathbf{6}}$ & $\boldsymbol{\lambda}_{\mathbf{7}}$ & $\boldsymbol{\lambda}_{\mathbf{8}}$ \\
\hline $\mathbf{1 0}$ & 3,215045955 & 6,292596434 & 9,427567258 & 12,56754708 & 15,70856552 & 18,84990441 & 21,99136804 & 25,132886950 \\
$\mathbf{5 0}$ & 3,467585253 & 6,329826163 & 9,438699854 & 12,57224968 & 15,71097386 & 18,85129819 & 21,99224569 & 25,133476400 \\
$\mathbf{1 0 0}$ & 3,721190961 & 6,375461338 & 9,452560641 & 12,57812054 & 15,71398272 & 18,85303997 & 21,99334274 & 25,134209970 \\
$\mathbf{2 0 0}$ & 4,112601888 & 6,463908152 & 9,480101377 & 12,58983775 & 15,71999529 & 18,85652210 & 21,99553618 & 25,135681680 \\
$\mathbf{5 0 0}$ & 4,883849037 & 6,709562392 & 9,561319893 & 12,62479524 & 15,73799190 & 18,86695697 & 22,00211273 & 25,140088566 \\
$\mathbf{1 0 0 0}$ & 5,678785297 & 7,067574639 & 9,692271528 & 12,68242292 & 15,76785029 & 18,88431020 & 22,01306060 & 25,147429051 \\
$\mathbf{1 5 0 0}$ & 6,234265490 & 7,378411599 & 9,818140700 & 12,73927864 & 15,79754069 & 18,90161591 & 22,02399227 & 25,154762587 \\
$\mathbf{2 0 0 0}$ & 6,671219285 & 7,654476911 & 9,939366318 & 12,79538616 & 15,82706529 & 18,91887441 & 22,03490765 & 25,162090591 \\
\hline \hline
\end{tabular}

\subsection{Clamped-Clamped Beam}

Table 7 shows that the frequency parameters for clamped-clamped beam.

Table 7: Frequency parameters for $\mathrm{C}-\mathrm{C}$ beam

\begin{tabular}{cccc|cccccc}
\hline \hline $\mathbf{K}_{\mathbf{0}}$ & \multicolumn{3}{c}{ Constant } & \multicolumn{3}{c|}{ Linear $(\alpha=0.2)$} & \multicolumn{3}{c}{ Parabolic $(\beta=0.2)$} \\
\hline & $\lambda_{1}$ & $\lambda_{2}$ & $\lambda_{3}$ & $\lambda_{1}$ & $\lambda_{2}$ & $\lambda_{3}$ & $\lambda_{1}$ & $\lambda_{2}$ & $\lambda_{3}$ \\
$\mathbf{1}$ & 4,7324 & 7,85372 & 10,9958 & 4,73217 & 7,85367 & 10,9958 & 4,73227 & 7,85369 & 10,9958 \\
{$[\mathbf{2 4}]^{*}$} & 4,730042 & 7,853203 & 10,99559 & - & - & - & - & - & \\
$\mathbf{1 0}$ & 4,75349 & 7,85836 & 10,9975 & 4,75116 & 7,85785 & 10,9973 & 4,75222 & 7,85805 & 10,9974 \\
$\mathbf{1 0 0}$ & 4,95039 & 7,90432 & 11,0144 & 4,92965 & 7,89925 & 11,0125 & 4,93914 & 7,90125 & 11,0132 \\
$\mathbf{1 0 0 0}$ & 6,22391 & 8,32512 & 11,179 & 6,11724 & 8,28151 & 11,1611 & 6,16646 & 8,29879 & 11,1677 \\
\hline \hline
\end{tabular}

* Squared results are tabulated in the reference for $\mathrm{K}_{0}=1$

\subsection{Cantilever Beam}

Frequency parameters for cantilever (C-F) beam can be seen in the Table 8 .

Table 8: Frequency parameters for cantilever (C-F) beam

\begin{tabular}{cccc|cccccc}
\hline \hline $\mathbf{K}_{\mathbf{0}}$ & \multicolumn{3}{c|}{ Constant } & \multicolumn{3}{c|}{ Linear $(\alpha=0.2)$} & \multicolumn{3}{c}{ Parabolic $(\beta=0.2)$} \\
\hline & $\lambda_{1}$ & $\lambda_{2}$ & $\lambda_{3}$ & $\lambda_{1}$ & $\lambda_{2}$ & $\lambda_{3}$ & $\lambda_{1}$ & $\lambda_{2}$ & $\lambda_{3}$ \\
$\mathbf{1}$ & 1,91192 & 4,69651 & 7,85527 & 1,90613 & 4,69622 & 7,85522 & 1,90708 & 4,69631 & 7,85524 \\
{$[\mathbf{2 4}]^{*}$} & 1,911926 & 4,696509 & 7,855272 & - & - & - & - & - & - \\
$\mathbf{1 0}$ & 2,1746 & 4,71808 & 7,85991 & 2,13427 & 4,71525 & 7,85936 & 2,14102 & 4,71611 & 7,85954 \\
$\mathbf{1 0 0}$ & 3,25578 & 4,9191 & 7,90584 & 3,13189 & 4,89398 & 7,90045 & 3,15299 & 4,90169 & 7,9022 \\
$\mathbf{1 0 0 0}$ & 5,64071 & 6,20825 & 8,32642 & 5,39828 & 6,08174 & 8,28005 & 5,43684 & 6,12325 & 8,29529 \\
\hline \hline
\end{tabular}

$*$ Squared results are tabulated in the reference for $\mathrm{K}_{0}=1$ 
The study covers the dynamic response of an Euler-Bernoulli beam in free vibration. The beam with constant cross-sectional area is supported along its length by variable Winkler elastic foundation. Winkler modeling is frequently applied to the beams and pipelines resting on an elastic soil. Such modeling introduces the elastic foundation by a set of mutually independent spring elements. In the present work, the elastic coefficient of the spring set is variable throughout the major axis of the beam. Three cases are studied: Constant, linear and parabolic variations. Also, three boundary cases are concerned: Simple support-Simple Support, Clamped-Clamped and Clamped-Free (cantilever) beams. Although the governing differential equations and boundary conditions are determined, they are not easily solvable. Hereof, corresponding studies which aim to solve the system equations with different approaches can be found in literature. In this study, the system equations are solved by Differential Transform Method, which is a succeeding and easy transformation technique. By solving the algebraic functions set, which are the transforms of differential equations, natural frequencies are obtained. The results are tabulated and compared with the former studies and a great accuracy to exact results is obtained.

\section{REFERENCES}

1. Gorbunov-Posadov, M.I., Malikova, T.A., The design of structures on an elastic foundation, Stroiizdat, Moscow, 1973.

2. K. Al-Hosani, S. Fadhil, A. El-Zafrany, Fundamental solution and boundary element analysis of thick plates on Winkler foundation. Computers and Structures 70 (1999), 325-336.

3. M. HetVenyi, Beams on elastic foundations. University of Michigan Press, Ann Arbor, 1961.

4. G. Gazetas, G. Mylonakis, Seismic soil-structure interaction: new evidence and emerging Issues. Geotechnical Special Publication 75 (1998), 1119-1174.

5. K.P. Soldatos, A. P. S. Selvadurai, Flexure of beams resting on hyperbolic elastic foundations. International Journal of Solids Structures 21 (1985), 373-388.

6. J.S. Smail, Large deflection response of annular plates on Pasternak foundations. International Journal of Solids Structures 27(1991), 1073-1084.

7. K. K. Raju, G. V. Rao, Effect of a non-linear elastic foundation on the mode shapes in stability and vibration problems of uniform columns/beams. Journal of Sound and Vibration 160 (1993), 369-371.

8. Y. S. Shih, B. T. Blotter, Non-linear vibration analysis of arbitrarily laminated thin rectangular plates on elastic foundations. Journal of Sound and Vibration 167(1993), 433-459.

9. D. Zhou, A General solution to vibrations of beams on variable Winkler elastic foundation. Computers \& Structures 47 (1993), 83-90.

10. L. Auersch, Dynamic interaction of various beams with the underlying soil -finite and infinite, half-space and Winkler models. European Journal of Mechanics A/Solids 27(2008), 933-958.

11. M. Eisenberger, J. Clastornik, Vibrations and buckling of a beam on a variable Winkler elastic foundation. Journal of Sound and Vibration 115 (1987), 233-241. 
12. U.S. Gupta, A.H. Ansari, S. Sharma, Buckling and vibration of polar orthotropic circular plate resting on Winkler foundation. Journal of Sound and Vibration 297 (2006), 457-476.

13. P. Ruge, C. Birk, A comparison of infinite Timoshenko and Euler-Bernoulli beam models on Winkler foundation in the frequency- and time-domain. Journal of Sound and Vibration 304 (2007), 932-947.

14. H.P. Lee, Dynamic response of a Timoshenko beam on a Winkler foundation subjected to a moving mass. Applied Acoustics 55 (1998), 203-215.

15. M. H. Huang, D.P. Thambiratnam, Deflection response of plate on Winkler foundation to moving accelerated loads, Engineering Structures 23 (2001), 1134-1141. 16. X. Ma, J.W. Butterworth, G.C. Clifton, Static analysis of an infinite beam resting on a tensionless Pasternak foundation. European Journal of Mechanics A/Solids 28 (2009), 697-703.

17. O. Civalek, Nonlinear analysis of thin rectangular plates on Winkler-Pasternak elastic foundations by DSC-HDQ methods. Applied Mathematical Modeling 31 (2007), 606-624.

18. Zhou, J.K., Differential transformation and its application for electrical circuits. Huazhong University Press, Wuhan, China, 1986.

19. A. Arıkoğlu, I. Özkol, Solution of boundary value problems for integro-differential equations by using differential transform method. Appl. Math. Comput. 168 (2005), $1145-1158$.

20. Ö. Özdemir, M.O. Kaya, Flapwise bending vibration analysis of a rotating tapered cantilever Bernoulli-Euler beam by differential transform method. Journal of Sound and Vibration 289 (2006), 413-420.

21. M.O. Kaya, Free vibration analysis of rotating Timoshenko beam by differential transform method. Aircr. Eng. Aerosp Technol. 78(3) (2006), 194-203.

22. Ö. Özdemir, M.O. Kaya, Flapwise bending vibration analysis of double tapered rotating Euler-Bernoulli beam by using the differential transform method. Meccanica 41(6) (2006), 661-670.

23. H. Liu, Y. Song, Differential transform method applied to high index differentialalgebraic equations, Appl. Math. Comput. 184 (2) (2007), 748-753.

24. B. Balkaya, M.O. Kaya, Analysis of the vibration of an elastic beam supported on elastic soil using the differential transform method. Meccanica 79 (2009), 135-146.

25. S.S. Rao, Mechanical Vibrations, $4^{\text {th }}$. Edition, Pearson Prentice Hall.

26. H. R. Öz, M. Pakdemirli, Two-to-one internal resonances in a shallow curved beam resting on an elastic foundation. Acta Mechanica, 185 (2006), 245-260

27. M. Eisenberger, Vibration frequencies for beams on variable one- and two-paramter elastic foundations. Journal of Sound and Vibrations 176(5) (1994), 577-584.

28. Ö. Civalek, Application of differential quadrature (DQ) and harmonic differential quadrature (HDQ) for buckling analysis of thin isotropic plates and elastic columns. Engineering Structures, 26 (2004), 171-186.

29. Ö. Civalek, Geometrically nonlinear dynamic analysis of doubly curved isotropic shells resting on elastic foundation by a combination of harmonic differential quadrature-finite difference methods. International Journal of Pressure Vessels and Piping 82 (2005), 470-479. 